\title{
Effect of Porphyromonas gingivalis infection on post-transcriptional regulation of the low-density lipoprotein receptor in mice
}

Haruna Miyazawa ${ }^{1,2}$, Koichi Tabeta $^{1 *}$, Sayuri Miyauchi, ${ }^{1,2}$, Yukari Aoki-Nonaka $^{1,2}$, Hisanori Domon? , Tomoyuki Honda ${ }^{1,2}$, Takako Nakajima ${ }^{1,3}$ and Kazuhisa Yamazaki ${ }^{1,2^{*}}$

\begin{abstract}
Background: Periodontal disease is suggested to increase the risk of atherothrombotic disease by inducing dyslipidemia. Recently, we demonstrated that proprotein convertase subtilisin/kexin type 9 (PCSK9), which is known to play a critical role in the regulation of circulating low-density lipoprotein (LDL) cholesterol levels, is elevated in periodontitis patients. However, the underlying mechanisms of elevation of PCSK9 in periodontitis patients are largely unknown. Here, we explored whether Porphyromonas gingivalis, a representative periodontopathic bacterium, -induced inflammatory response regulates serum PCSK9 and cholesterol levels using animal models.

Methods: We infected C57BL/6 mice intraperitoneally with Porphyromonas gingivalis, a representative strain of periodontopathic bacteria, and evaluated serum PCSK9 levels and the serum lipid profile. PCSK9 and LDL receptor (LDLR) gene and protein expression, as well as liver $X$ receptors (Lxrs), inducible degrader of the LDLR (Idol), and sterol regulatory element binding transcription factor (Srebf)2 gene expression, were examined in the liver.

Results: P. gingivalis infection induced a significant elevation of serum PCSK9 levels and a concomitant elevation of total and LDL cholesterol compared with sham-infected mice. The LDL cholesterol levels were significantly correlated with PCSK9 levels. Expression of the Pcsk9, Ldlr, and Srebf2 genes was upregulated in the livers of the $P$. gingivalis-infected mice compared with the sham-infected mice. Although Pcsk9 gene expression is known to be positively regulated by sterol regulatory element binding protein (SREBP)2 (human homologue of Srebf2), whereas Srebf2 is negatively regulated by cholesterol, the elevated expression of Srebf2 found in the infected mice is thought to be mediated by $P$. gingivalis infection.
\end{abstract}

Conclusions: P. gingivalis infection upregulates PCSK9 production via upregulation of Srebf2, independent of cholesterol levels. Further studies are required to elucidate how infection regulates Srebf2 expression and subsequently influences lipid metabolism.

Keywords: PCSK9, LDL cholesterol, Periodontitis, Chronic inflammation

\footnotetext{
* Correspondence: koichi@dent.niigata-u.ac.jp; kaz@dent.niigata-u.ac.jp

${ }^{1}$ Center for Transdisciplinary Research, Niigata University, 5274 Gakkocho 2-ban-cho, Chu-o-ku, Niigata 951-8514, Japan

2Laboratory of Periodontology and Immunology, Division of Oral Science for Health Promotion, Niigata University Graduate School of Medical and Dental Sciences, 5274 Gakkocho 2-ban-cho, Chu-o-ku, Niigata 951-8514, Japan

Full list of author information is available at the end of the article
} 


\section{Background}

Periodontitis is associated with atherosclerotic vascular disease, as it induces dyslipidemia. It has been found that periodontitis decreases HDL cholesterol and increases LDL cholesterol levels. It has also been reported that the presence of periodontal pockets was positively associated with higher LDL and total cholesterol in humans [1]. Löesche et al. showed that the levels of total and LDL cholesterol were significantly higher in 50- to 60-year-old patients with moderate periodontitis compared with ageand sex-matched controls [2]. Furthermore, intensive periodontal therapy consisting of subgingival mechanical debridement with adjunctive local delivery of minocycline significantly decreased total and LDL cholesterol compared with baseline levels [3]. The mechanisms underlying the elevation of LDL cholesterol levels in periodontitis patients have not yet been elucidated.

Plasma cholesterol levels are regulated by the LDL receptor (LDLR). The number of LDLRs expressed on the hepatocyte surface is the primary determinant of plasma cholesterol levels and is therefore strictly regulated. Transcription of the LDLR gene is controlled by cellular cholesterol levels through the sterol regulatory elementbinding protein (SREBP) [4] and Liver X receptors (LXRs) [5]. Additionally, post-transcriptional regulation of LDLR expression is a major determinant of lipoprotein metabolism.

Proprotein convertase subtilisin/kexin type 9 (PCSK9) post-transcriptionally promotes the degradation of LDLRs in hepatocytes [6]. PCSK9 is mainly expressed in the liver, intestine, and kidney, and it is secreted into the plasma [7]. This protein does not directly degrade LDLRs but binds to LDLRs at epidermal growth factorlike repeats. This binding decreases LDLR recycling to the cell surface and promotes lysosomal degradation [8], which results in decreased numbers of LDLRs and increased plasma LDL levels. We have recently shown that serum levels of PCSK9 are significantly increased in periodontitis patients compared with periodontally healthy subjects [9]. In addition to PCSK9, the inducible degrader of the LDL receptor (Idol) has been shown to play an important role in the post-transcriptional regulation of LDLRs. Zelcer et al. [5] found that LDLR expression is controlled by the LXR-Idol axis as LXR induces Idol, which is an E3 ubiquitin ligase that triggers LDLR degradation.

It is well-recognized that infection and inflammation induce marked changes in lipid and lipoprotein metabolism [10]. However, there is little information about the effects of infection on PCSK9 or Idol levels and subsequent expression of LDLR and cholesterol levels. Only one study has shown that inflammatory stimuli, such as lipopolysaccharide or zymosan, markedly increased hepatic PCSK9 mRNA expression, resulting in decreased
LDLR protein expression [11]. Therefore, in the present study, we analyzed the effects of infection with Porphyromonas gingivalis, a representative strain of periodontopathic bacteria, on the regulation of PCSK9 and subsequently expression of LDLR in the liver.

\section{Results}

\section{Serum SAA and PCSK9 levels}

Systemic infection with $P$. gingivalis induced a significant elevation of serum amyloid A (SAA) levels. The PCSK9 levels in the infected mice were significantly higher than in the sham-infected mice (Figure 1).

\section{Plasma lipid profiles}

The total and LDL cholesterol levels were significantly higher in the $P$. gingivalis-infected mice compared with the sham-infected mice. In contrast, HDL cholesterol levels tended to be lower in the $P$. gingivalis-infected mice than the sham-infected mice. There was no difference in triglyceride levels between the $P$. gingivalisinfected mice and sham-infected mice (Figure 2).

\section{Relationship between serum PCSK9 and LDL cholesterol levels}

Similar to a previous study by our group that demonstrated a close relationship between PCSK9 and LDL cholesterol, we found a significant correlation between PCSK9 levels and LDL cholesterol levels after $P$. gingivalis infection (Figure 3).

However, no correlations were observed between PCSK9 levels and total cholesterol levels, HDL cholesterol levels, or triglyceride levels (data not shown).

\section{PCSK9 and LDLR gene and protein expression in the liver} There was a significant increase in the expression of PCSK9 and LDLR (Figure 4A) in the infected mice compared to the sham-infected mice. However, there was a much larger increase in gene expression observed for PCSK9 than LDLR. Although Pcsk9 gene expression was increased, the levels of mature PCSK9 protein in the liver of the $P$. gingivalis-infected mice were similar to those of the sham-infected mice. Pro-PCSK9 protein levels were even lower in the $P$. gingivalis-infected mice compared with the sham-infected mice. In contrast to the gene expression results, the protein levels of LDLR were significantly lower in the livers of the $P$. gingivalisinfected mice compared with the sham-infected mice (Figure $4 \mathrm{~B}$ and $\mathrm{C}$ ).

\section{Changes in Srebf2, Lxrs, and Idol gene expression}

SREBP2 (Srebf2 in mice) is a major activator of PCSK9 signaling. Therefore, the effect of $P$. gingivalis infection on PCSK9 expression was analyzed. As shown in Figure 5A, Srebf2 expression was significantly upregulated in the 

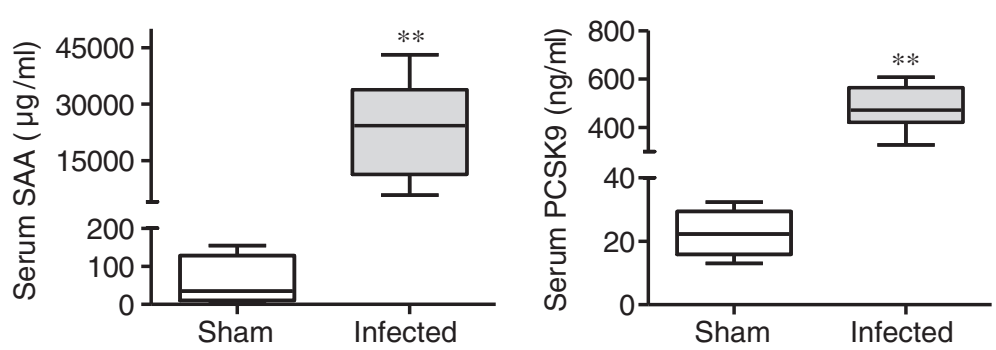

Figure 1 Effects of $P$. gingivalis infection on serum levels of serum amyloid A (SAA) and PCSK9 ( $N=5$ in the sham-infected group; $N=6$ in the infected group). All samples were analyzed in duplicate for each condition. Box plots represent medians, with the $25^{\text {th }}$ and $75^{\text {th }}$ percentiles shown as boxes and the $10^{\text {th }}$ and $90^{\text {th }}$ percentiles as whiskers. Significant differences were observed between the infected group and the sham-infected group (** $P<0.01$, Mann-Whitney U-test).

P. gingivalis-infected mice compared with the shaminfected mice.

Because of the important role of Idol in the posttranscriptional regulation of LDLR expression, the effects of $P$. gingivalis infection on Idol gene expression and the gene expression of Lxrs, a regulator of the Idol gene, were examined. Idol gene expression was slightly, but significantly suppressed in the $P$. gingivalis-infected mice compared with the sham-infected mice (Figure 5B). However, no differences in $L x r \alpha$ or $L x r \beta$ gene expression were observed, although the expression of both genes tended to be higher in the $P$. gingivalis-infected mice (Figure 5C).

\section{Discussion}

Given that humans with loss-of-function mutations in the PCSK9 gene [12] and mice lacking Pcsk9 expression exhibit significantly reduced circulating LDL cholesterol levels [13], whereas activating mutations in the PCSK9 gene result in severe familial hypercholesterolemia [14] accompanied by increased cardiovascular risk, PCSK 9 is considered at present to be an important therapeutic target for combating hypercholesterolemia [15]. In addition, other factors that elevate circulating PCSK9 levels could be considered to be risk factors for coronary heart disease (CHD). A previous study by our group demonstrated that periodontitis, a chronic inflammatory disease induced by a group of periodontopathic bacteria, could elevate serum PCSK9 levels [9]. However, the underlying mechanisms by which periodontal infection affects PCSK9 levels and the subsequent alterations of the plasma lipid profile are not known.

It is well-known that infection and inflammation induce an acute phase response, which leads to multiple alterations in lipid and lipoprotein metabolism [10]. We have
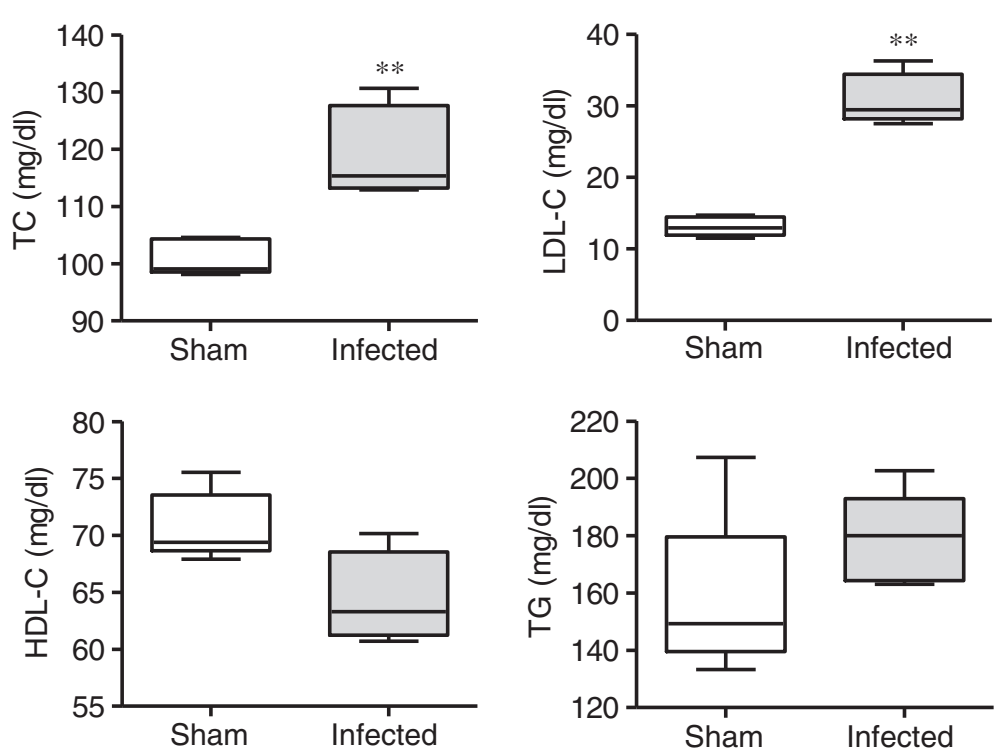

Figure 2 Effects of $\boldsymbol{P}$. gingivalis infection on the plasma lipid profile ( $\mathbf{N}=\mathbf{5}$ in each group). Box plots represent medians, with the $25^{\text {th }}$ and $75^{\text {th }}$ percentiles as boxes and the $10^{\text {th }}$ and $90^{\text {th }}$ percentiles as whiskers. Total and LDL cholesterol levels were significantly higher, whereas HDL cholesterol levels tended to be lower in the $P$. gingivalis-infected mice (** $P<0.01$, Mann-Whitney U-test). 


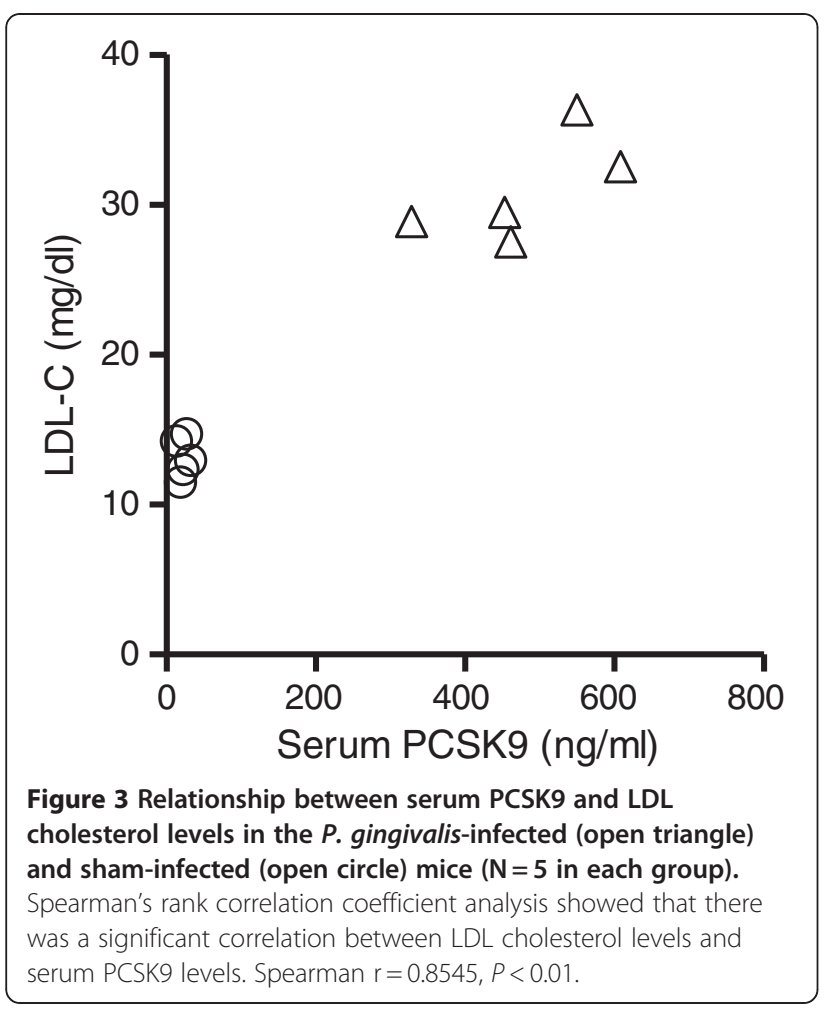

shown that chronic oral infection with $P$. gingivalis induced downregulation of HDL cholesterol by suppressing Liver $\mathrm{X}$ receptors and their target gene $A b c a 1$ in C57BL/6 mice and ApoE-deficient C57BL/6.KOR-Apoe ${ }^{\text {shl }}$ (B6.Apoeshl) mice [16]. Furthermore, as in human periodontitis patients, the LDL cholesterol levels were elevated in P. gingivalis-infected B6.Apoeshl mice. However, the molecular basis for the elevation of LDL cholesterol was not analyzed in this experimental setting.

This study is the first to show that infection induces a significant increase in circulating PCSK9 levels and a concomitant decrease of LDLR levels in mice. Furthermore, there was a significant correlation found between LDLR gene expression in the liver and the serum levels of PCSK9. In this study, infection was induced via intraperitoneal, rather than oral, bacterial inoculation. Although oral infection can mimic human periodontal disease, and it is clear that oral infection with $P$. gingivalis does, in fact, affect Pcsk 9 gene expression in the liver (data not shown), subsequent change in lipid metabolism can be easily observed in peritoneal infection model.

Pcsk9 gene expression was significantly upregulated in the liver of the $P$. gingivalis-infected mice. Previous studies have demonstrated that Pcsk9 and Ldlr gene transcription is regulated by sterol regulatory elementbinding proteins (SREBPs) $[17,18]$. Consistent with these studies, Srebf2 expression was found to be significantly upregulated in the livers of the $P$. gingivalis-infected mice compared with the sham-infected mice. Srebf2 expression is downregulated by cholesterol and upregulated by statin treatment. As total and LDL cholesterol were elevated in the $P$. gingivalis-infected mice, Srebf 2 is likely to be induced by this infection. Although the mechanisms by which infection and/or inflammation induce the upregulation of Srebf2 are not clear, our study suggests that additional mechanisms related to infection induce hyperlipidemia during infection.

Recently, another transcription factor, known as hepatocyte nuclear factor-1 $\alpha$ (HNF1 $\alpha$ ), was reported to be involved in the transcriptional regulation of the Pcsk9 gene in mammalian HepG2 cells [19]. However, it has not been reported whether Pcsk9 is activated by HNF1 $\alpha$ in mice. Therefore, other mechanisms may regulate Pcsk9 expression during infection; further studies will be needed to answer this question.

In contrast to the gene expression results, the level of mature PCSK9 protein in the liver was unchanged in the $P$. gingivalis-infected mice compared to the shaminfected mice, whereas the levels of PCSK9 proprotein were slightly reduced in the $P$. gingivalis-infected mice. Because we failed to observe the enhanced transcription of PCSK9 in other organ specimen such as liver, kidney, jejulium, ilenium, aolta, and spleen (data not shown), we speculate that secreted PCSK9 immediately infiltrate into circulation and result in the elevation of PCSK9 levels higher in the P. gingivalis-infected mice. Recently, it has become evident that domain interactions are important for the regulation of PCSK9 protein secretion [20]. However, it remains to be elucidated how infection affects PCSK9 protein secretion.

Similar to Pcsk9, Ldlr is upregulated in the livers of the $P$. gingivalis-infected mice compared with the shaminfected mice, although to a much lesser degree. Because LDLR expression is also positively regulated by SREBP2 [18], upregulation of $L d l r$ could be an effect of the infection. Contrary to what was observed regarding Ldlr gene expression, LDLR protein expression in the liver of the $P$. gingivalis-infected mice was significantly reduced compared with the sham-infected mice. Although the $L d l r$ gene expression levels in the P. gingivalis-infected mice were 2 times higher compared with the shaminfected mice, the expression of LDLR protein was decreased, possibly because PCSK9 serum levels were increased more than 20 fold in the infected mice.

In addition to PCSK9, LDLR expression is controlled by the LXR-Idol axis, in which LXR induces Idol, an E3 ubiquitin ligase that triggers LDLR degradation [5]. The importance of Idol in the regulation of LDLR expression is increasingly being recognized [21]. Therefore, the affect of $P$. gingivalis infection on Idol gene expression was analyzed. Although the expression of the Idol gene was significantly down-regulated in the $P$. gingivalis-infected mice, the difference in Idol gene expression between the 
A.

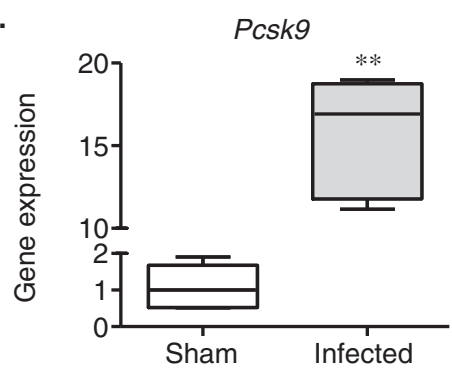

B.

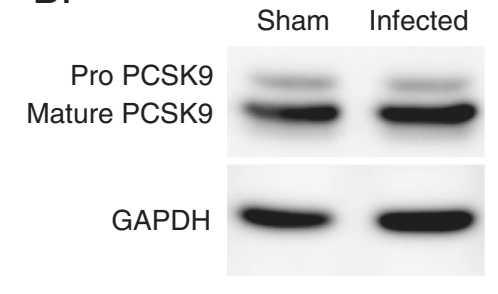

C.

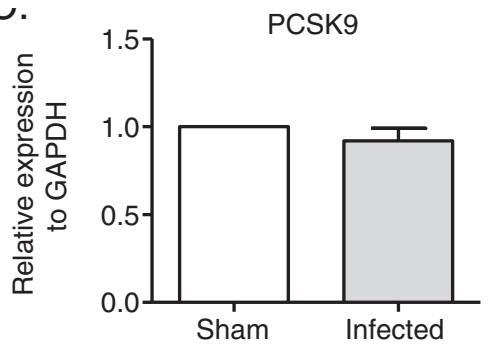

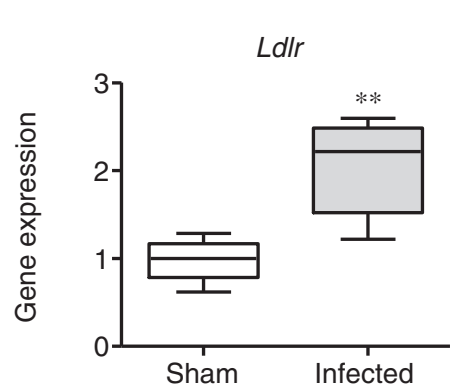
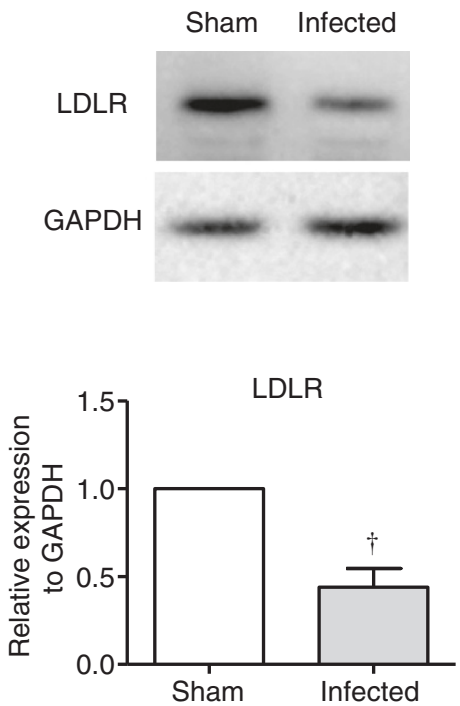

Figure 4 Effect of $P$. gingivalis infection on PCSK9 and LDLR gene and protein expression in the liver. Total RNA and protein were extracted from the $P$. gingivalis-infected and sham-infected mice, and the samples were analyzed by quantitative RT-PCR or western blotting. Pcsk9 and Ldlr gene expression was significantly higher in the $P$. gingivalis-infected mice compared with the sham-infected mice ${ }^{* *} P<0.01$, Mann-Whitney U-test). The PCSK9 protein levels of were unchanged in the $P$. gingivalis-infected mice compared with the sham-infected mice. LDLR protein levels were significantly lower in the $P$. gingivalis-infected mice compared with the sham-infected mice $\left({ }^{\dagger} P<0.05\right.$, unpaired t-test).

infected and the sham-infected mice was smaller than what was observed for Pcsk9. In addition, the expression of Lxrs did not differ between the P. gingivalis-infected mice and the sham-infected mice. Therefore, it is possible that $P$. gingivalis infection does not affect LDLR expression by downregulating Idol in this experimental model.

Our study supports the previously described notion that bacterial infections alter the plasma lipid profile based on the novel finding that $P$. gingivalis infection elevates PCSK9 expression in the liver by increasing Srebf2 expression through a currently unidentified mechanism. The effects of infection and inflammation on lipid and lipoprotein metabolism are complex, and contrasting results are often demonstrated between rodents and primates [10]. Our animal study model has potential limitations considering the characteristics of chronic inflammation in human periodontitis, because peritoneal infection in mice induces robust acute immune response. There may be other proteins that are involved in the regulation of
PCSK9 by the mechanisms related to inflammation. Further studies will be required to gain insight into the role of PCSK9 in the dyslipidemia that accompanies infections and chronic inflammatory diseases, such as periodontitis.

\section{Conclusions}

We found that $P$. gingivalis infection upregulates PCSK9 production via upregulation of Srebf2, independent of cholesterol levels resulting elevation of LDL cholesterol. Further studies are required to elucidate how infection regulates Srebf2 expression and subsequently influences lipid metabolism.

\section{Methods}

\section{Mice}

All experiments were performed in accordance with the Regulations and Guidelines on Scientific and Ethical Care and Use of Laboratory Animals of the Science Council of Japan, enforced on June 1, 2006 and approved 


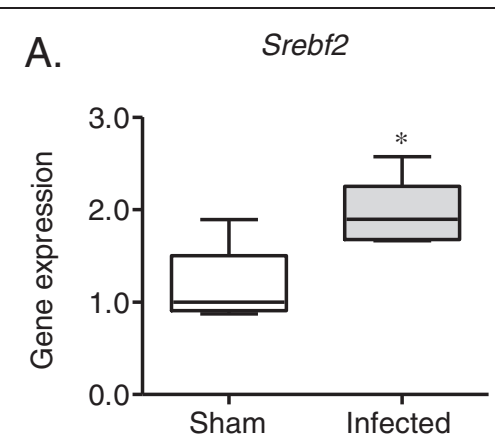

B. $\quad$ Idol
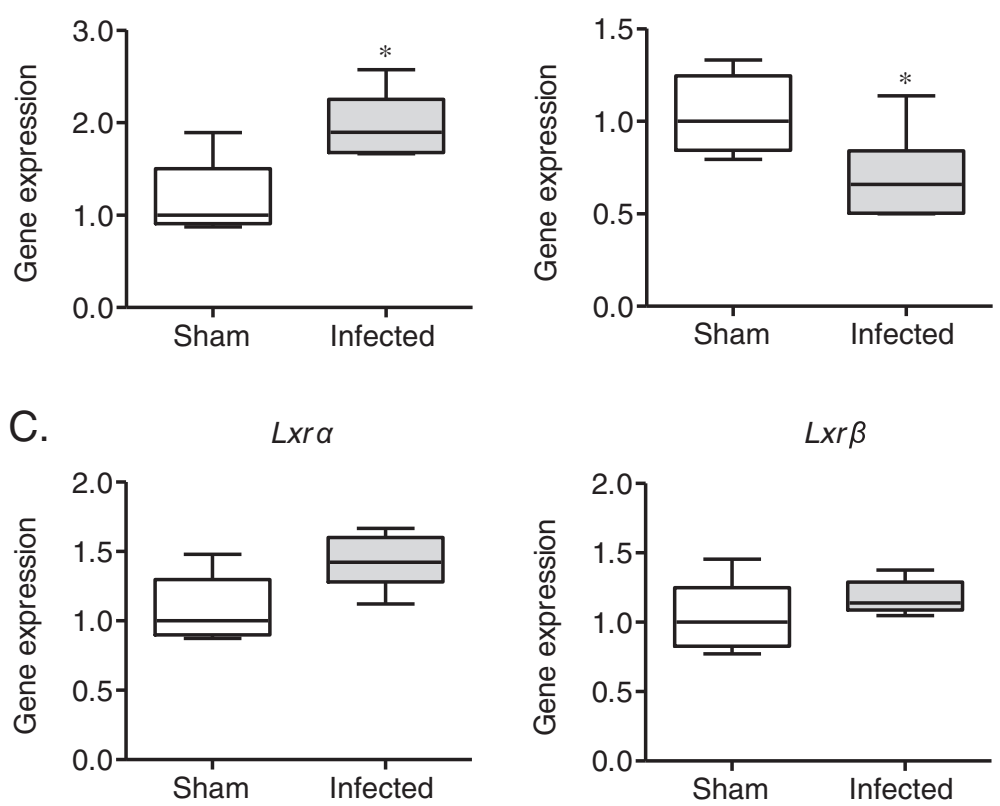

Figure 5 Comparison of the relative gene expression levels in the liver between the $P$. gingivalis-infected mice and the sham-infected mice ( $N=5$ in the sham-infected group, $N=6$ in the infected group). The relative mRNA levels for the investigated genes were normalized to the relative glyceraldehyde-3-phosphate dehydrogenase (GAPDH) mRNA levels. The box plots represent medians, with the $25^{\text {th }}$ and $75^{\text {th }}$ percentiles being depicted as boxes and the $10^{\text {th }}$ and $90^{\text {th }}$ percentiles presented as whiskers. Significant differences were observed for Srebf2 and Idol (* $P<0.05$, Mann-Whitney U-test).

by the Institutional Animal Care and Use Committee at Niigata University (permit number 231-1). Six-week-old male C57BL/6 mice were obtained from Japan SLC, Inc. (Shizuoka, Japan). The mice were maintained under pathogen-free conditions and were fed regular chow and sterile water until they were infected.

\section{Bacterial cultures and infection}

P. gingivalis strain W83 was cultured in modified Gifu anaerobic medium (GAM) broth (Nissui, Tokyo, Japan) in an anaerobic jar (Becton Dickinson Microbiology Systems, Cockeysville, MD) in the presence of an AnaeroPack ${ }^{\mathrm{TM}}$ (Mitsubishi Gas Chemical Co. Inc., Tokyo, Japan) for 48 hours at $37^{\circ} \mathrm{C}$. Bacterial suspensions were prepared in $\mathrm{Mg}^{2+} / \mathrm{Ca}^{2+}$-free phosphate-buffered saline (PBS) using spectrophotometry to establish growth curves. The number of CFUs was standardized by measuring the optical density at $600 \mathrm{~nm}$. The mice in the experimental groups were administered a single intraperitoneal (i.p.) inoculum of $10^{9} \mathrm{CFU}$ in $0.2 \mathrm{ml}$ of sterile phosphate-buffered saline (PBS), pH 7.2. Control mice were inoculated with PBS alone. Mice were euthanized $16 \mathrm{hrs}$ after infection and then analyzed.

\section{Real-time PCR}

Total RNA was isolated from the liver using TRIzol $^{\mathrm{TM}}$ (Life Technologies, Carlsbad, CA) according to the manufacturer's instructions. Next, aliquots of RNA were reverse transcribed to produce cDNA using random primers (Takara Bio Inc., Shiga, Japan) and M-MLV reverse transcriptase (Life Technologies, Carlsbad, CA). Specific primers and probes for real-time PCR were purchased from Applied Biosystems (Foster City, CA). TaqMan Gene Expression Assays (Applied Biosystems) were performed in 20- $\mu$ l reactions containing $900 \mathrm{nM}$ primers and $250 \mathrm{nM}$ probe using an ABI PRISM 7900HT Sequence Detection System (Applied Biosystems). The reaction conditions involved a 10-minute incubation at $95^{\circ} \mathrm{C}$ followed by 40 cycles of a two-step amplification procedure consisting of annealing/extension at $60^{\circ} \mathrm{C}$ for 1 minute and denaturation for 15 seconds at $95^{\circ} \mathrm{C}$. ABI PRISM SDS 2.0 software (Applied Biosystems) was used to analyze the standards and quantify the data. The relative quantity of each mRNA sequence was normalized to the relative quantity of glyceraldehyde-3-phosphate dehydrogenase (GAPDH) mRNA.

\section{Western blotting}

The livers of the mice were dissected into small pieces, and protein was extracted using T-PER Tissue Protein Extraction Reagent (Thermo Scientific, Rockford, IL) supplemented with a Halt Protease Inhibitor Cocktail Kit (Thermo Scientific) and Halt Phosphatase Inhibitor 
Cocktail (Thermo Scientific) according to the manufacturer's instructions. Cell debris was pelleted by centrifugation at $10,000 \times g$ for 5 minutes at $4^{\circ} \mathrm{C}$. The protein concentration in the supernatant was determined using the Pierce BCA Protein Assay kit (Thermo Scientific) according to the manufacturer's instructions.

Thirty micrograms of each sample were solubilized in SDS sample buffer, separated by SDS-PAGE, and transferred to a polyvinylidene difluoride membrane (Immobilon-P; Millipore Co., Bedford, MA). The samples were subsequently subjected to western blotting with rabbit anti-mouse PCSK9 (Abcam, Cambridge, UK) or rabbit anti-mouse LDLR (Abcam) primary antibodies followed by $\mathrm{ECL}^{\mathrm{TM}}$ Peroxidase labeled anti-rabbit secondary antibodies (GE Healthcare, Buckinghamshire, UK). The blots were developed with ECL using Lumi Vision PRO 400EX (Aisin, Aichi, Japan). The membranes were then washed 3 times with wash buffer (Tris-buffered saline containing $0.1 \%$ Tween-20 and 0.5\% BSA) and reprobed with goat anti-mouse GAPDH antibodies (Santa Cruz Biotechnology, Santa Cruz, CA), as described above. Horseradish peroxidase-labeled donkey anti-goat IgG (Santa Cruz) was used as secondary antibody for reprobing. The intensity of the signal was quantified with Scion Image 4.02 computer software. The intensity of each protein band was normalized to the intensity of GAPDH.

\section{Serum PCSK9, SAA, and lipoprotein levels}

SAA and PCSK9 were measured using commercially available ELISA kits (Tridelta Development Ltd., Kildare, UK and R \& D Systems, Minneapolis, MN, respectively) in serum collected prior to euthanasia. Serum cholesterol and triglyceride profiles were analyzed at Skylight Biotech Inc. (Akita, Japan).

\section{Statistical analyses}

The differences in the examined gene expression and biochemical parameters between the infected and control mice were analyzed using the Mann-Whitney Utest. Linear correlations were obtained using Spearman's rank correlation coefficient analysis. Unpaired t-tests were used for densitometric analysis. The statistical analyses were performed using standard statistical software (GraphPad Prism, GraphPad Software Inc., La Jolla, CA and StatView J-5.0 application program, SAS Institute Inc., Cary, NC). $P<0.05$ was considered to be statistically significant.

\section{Abbreviations}

PCSK9: Proprotein convertase subtilisin/kexin type 9; LDL: Low-density lipoproteins; HDL: High-density lipoproteins; LDLR: LDL receptor; LXR: Liver X receptor; Idol: Inducible degrader of the LDLR; Srebf2: Sterol regulatory element binding transcription factor2; SREBP: Sterol regulatory element binding protein; LPS: Lipopolysaccharide; B6.Apoeshl: C57BL/6.KOR-Apoe $e^{\text {shl; }}$ HNF1a: Hepatocyte nuclear factor-1.

\section{Competing interests}

The authors declare that they have no competing interests.

\section{Authors' contributions}

$K T, T N$, and KY designed the study; HM, SM, YA-N, HD and TM performed the experiments; KT, TN and KY wrote the paper. All authors read and approved the final manuscript.

\section{Acknowledgements}

This study was supported by the Japan Society for the Promotion of Science (23390476 and 23659974 for KY, 20295426 for KT, and 21390555 for TN), the Young Researcher Overseas Visits Program for Vitalizing Brain Circulation (S2203), and the Promotion of Niigata University Research Project.

\section{Author details}

${ }^{1}$ Center for Transdisciplinary Research, Niigata University, 5274 Gakkocho 2-ban-cho, Chu-o-ku, Niigata 951-8514, Japan. 'Laboratory of Periodontology and Immunology, Division of Oral Science for Health Promotion, Niigata University Graduate School of Medical and Dental Sciences, 5274 Gakkocho 2-ban-cho, Chu-o-ku, Niigata 951-8514, Japan. ${ }^{3}$ General Dentistry and Clinical Education Unit, Niigata University Medical and Dental Hospital, 5274

Gakkocho 2-ban-cho, Chu-o-ku, Niigata 951-8514, Japan.

Received: 24 May 2012 Accepted: 14 September 2012

Published: 19 September 2012

\section{References}

1. Katz J, Flugelman MY, Goldberg A, Heft M: Association between periodontal pockets and elevated cholesterol and low density lipoprotein cholesterol levels. J Periodontol 2002, 73:494-500.

2. Losche W, Karapetow F, Pohl A, Pohl C, Kocher T: Plasma lipid and blood glucose levels in patients with destructive periodontal disease. $J$ Clin Periodontol 2000, 27:537-541.

3. D'Aiuto F, Nibali L, Parkar M, Suvan J, Tonetti MS: Short-term effects of intensive periodontal therapy on serum inflammatory markers and cholesterol. J Dent Res 2005, 84:269-273.

4. Yokoyama C, Wang X, Briggs MR, Admon A, Wu J, Hua X, Goldstein JL, Brown MS: SREBP-1, a basic-helix-loop-helix-leucine zipper protein that controls transcription of the low density lipoprotein receptor gene. Cell 1993, 75:187-197.

5. Zelcer N, Hong C, Boyadjian R, Tontonoz P: LXR regulates cholesterol uptake through Idol-dependent ubiquitination of the LDL receptor. Science 2009, 325:100-104.

6. Lambert G, Charlton F, Rye KA, Piper DE: Molecular basis of PCSK9 function. Atherosclerosis 2009, 203:1-7

7. Seidah NG, Benjannet S, Wickham L, Marcinkiewicz J, Jasmin SB, Stifani S, Basak A, Prat A, Chretien M: The secretory proprotein convertase neural apoptosis-regulated convertase 1 (NARC-1): liver regeneration and neuronal differentiation. Proc Natl Acad Sci US A 2003, 100:928-933.

8. Zhang DW, Lagace TA, Garuti R, Zhao Z, McDonald M, Horton JD, Cohen JC, Hobbs $\mathrm{HH}$ : Binding of proprotein convertase subtilisin/kexin type 9 to epidermal growth factor-like repeat A of low density lipoprotein receptor decreases receptor recycling and increases degradation. $J$ Biol Chem 2007, 282:18602-18612.

9. Miyazawa H, Honda T, Miyauchi S, Domon H, Okui T, Nakajima T, Tabeta K, Yamazaki K: Increased serum PCSK9 concentrations are associated with periodontal infection but do not correlate with LDL cholesterol concentration. Clin Chim Acta 2012, 413:154-159.

10. Khovidhunkit W, Kim MS, Memon RA, Shigenaga JK, Moser AH, Feingold KR, Grunfeld C: Effects of infection and inflammation on lipid and lipoprotein metabolism: mechanisms and consequences to the host. J Lipid Res 2004, 45:1169-1196.

11. Feingold KR, Moser AH, Shigenaga JK, Patzek SM, Grunfeld C: Inflammation stimulates the expression of PCSK9. Biochem BiophysRes Commun 2008, 374:341-344.

12. Cohen J, Pertsemlidis A, Kotowski IK, Graham R, Garcia CK, Hobbs HH: Low $\mathrm{LDL}$ cholesterol in individuals of African descent resulting from frequent nonsense mutations in PCSK9. Nat Genet 2005, 37:161-165.

13. Rashid S, Curtis DE, Garuti R, Anderson NN, Bashmakov Y, Ho YK, Hammer RE, Moon YA, Horton JD: Decreased plasma cholesterol and 
hypersensitivity to statins in mice lacking Pcsk9. Proc Natl Acad Sci U S A 2005, 102:5374-5379.

14. Abifadel M, Varret M, Rabes JP, Allard D, Ouguerram K, Devillers M, Cruaud C, Benjannet S, Wickham L, Erlich D, et al: Mutations in PCSK9 cause autosomal dominant hypercholesterolemia. Nat Genet 2003, 34:154-156.

15. Lambert G, Krempf M, Costet P: PCSK9: a promising therapeutic target for dyslipidemias? Trends Endocrinol Metab 2006, 17:79-81.

16. Maekawa T, Takahashi N, Tabeta K, Aoki Y, Miyashita H, Miyauchi S, Miyazawa H, Nakajima T, Yamazaki K: Chronic Oral Infection with Porphyromonas gingivalis accelerates atheroma formation by shifting the lipid profile. PLOS ONE 2011, 6:e20240.

17. Dubuc G, Chamberland A, Wassef H, Davignon J, Seidah NG, Bernier L, Prat A: Statins upregulate PCSK9, the gene encoding the proprotein convertase neural apoptosis-regulated convertase-1 implicated in familial hypercholesterolemia. Arterioscler Thromb Vasc Biol 2004, 24:1454-1459.

18. Jeong HJ, Lee HS, Kim KS, Kim YK, Yoon D, Park SW: Sterol-dependent regulation of proprotein convertase subtilisin/kexin type 9 expression by sterol-regulatory element binding protein-2. J Lipid Res 2008, 49:399-409.

19. Li H, Dong B, Park SW, Lee HS, Chen W, Liu J: Hepatocyte nuclear factor 1alpha plays a critical role in PCSK9 gene transcription and regulation by the natural hypocholesterolemic compound berberine. J Biol Chem 2009, 284:28885-28895.

20. Du F, Hui Y, Zhang M, Linton MF, Fazio S, Fan D: Novel domain interaction regulates secretion of proprotein convertase subtilisin/kexin type 9 (PCSK9) protein. J Biol Chem 2011, 286:43054-43061.

21. Sorrentino V, Zelcer N: Post-transcriptional regulation of lipoprotein receptors by the E3-ubiquitin ligase inducible degrader of the lowdensity lipoprotein receptor. Curr Opin Lipidol 2012, 23(3):213-219.

doi:10.1186/1476-511X-11-121

Cite this article as: Miyazawa et al:: Effect of Porphyromonas gingivalis infection on post-transcriptional regulation of the low-density

lipoprotein receptor in mice. Lipids in Health and Disease 2012 11:121.

\section{Submit your next manuscript to BioMed Central and take full advantage of:}

- Convenient online submission

- Thorough peer review

- No space constraints or color figure charges

- Immediate publication on acceptance

- Inclusion in PubMed, CAS, Scopus and Google Scholar

- Research which is freely available for redistribution 